\title{
PREPARATION AND EVALUATION OF PIROXICAM - PLURONIC SOLID DISPERSIONS
}

\author{
M. Fathy and M. El-Badry
}

Department of Pharmaceutics, Faculty of Pharmacy, Assiut University, Assiut, Egypt

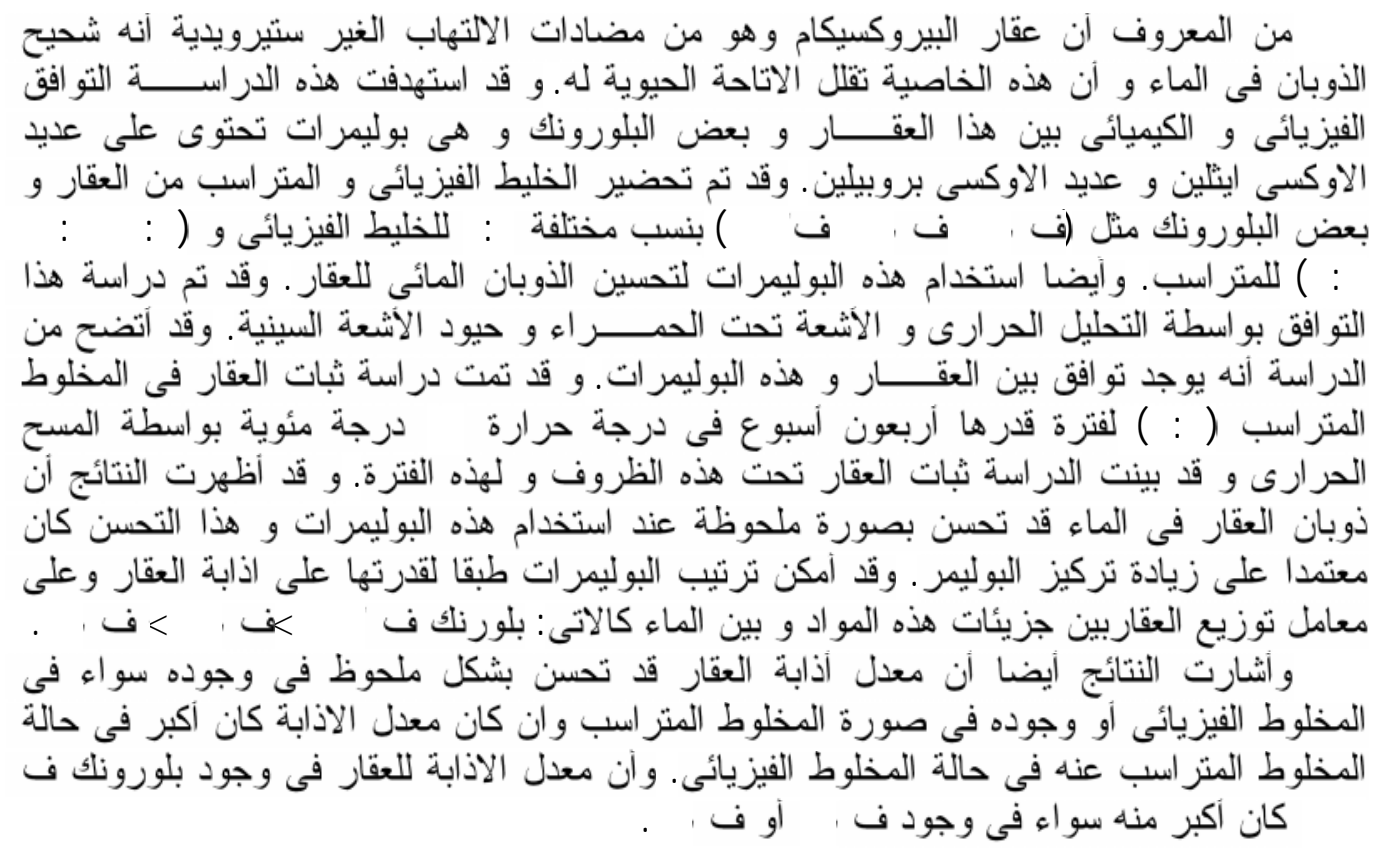

With an objective of enhancing the dissolution rate of piroxicam (PXM), solid dispersions $(S D s)$ of piroxicam were prepared using different grades of pluronic F-68 (Pl. F-68), pluronic F-98 (Pl. F-98), and pluronic F-127 (Pl. F-127) adopting the solvent evaporation technique. The solid dispersions were prepared at different (drug: polymer) ratio (1:1, 1:3 and 1:5). Piroxicam- pluronic solid dispersions were investigated by inferared spectroscopy (IR), $x$-ray diffraction $(X R D)$ and differential scanning calorimetry (DSC). The IR spectra prevailed no chemical interaction between the drug and copolymer. The powder $x$-ray diffraction (XRD) patterns and differential scanning calorimetry (DSC) thermograms demonstrated that piroxicam was existed in a solid solution state in the SD. Phase diagram for solid dispersion of piroxicam in pluronic F-98 indicated that the solid solution of piroxicam was obtained at a concentration of PXM less than $35 \%$ in the SD. The stability study was carried out for the solid dispersion prepared at 1:1 (piroxicam/ Pl. F-98) at $40^{\circ}$ for 40 weeks prevailed that; the drug is stable during the storage period in the dispersion. The solubility study of piroxicam was carried out at different concentrations of pluronics, also the dissolution rate of the drug was improved either by physical mixture or the solid dispersions.

\section{INTRODUCTION}

Currently, in the field of pharmaceutical technology, great efforts are being directed towards the solving problems related to poorwater solubility and poor bioavailability of drugs. This trend of working has led to development of different techniques. Sekiguchi and $\mathrm{Obi}^{1}$ first introduced the concept of solid dispersions as a drug delivery system, where urea is used as the carrier for sulphathiazole as a poorly water-soluble drug. Subsequent research works were done to improve the dissolution and bioavailability of poorly 
soluble drugs. ${ }^{2-4}$ Solid dispersions can be obtained by three procedures: by melting, by using solvents or by combined process including melting and solvents. ${ }^{5}$

Piroxicam is a non-steroid antiinflammatory compound with analgesic and antipyretic effects, used for the treatment of rheumatoid arthritis, ankylosing spondylitis, acute gout, acute musculoskelatal disorders, primary dysmenorrhea, osteoarthritis and traumatic contusions. ${ }^{6,7}$ The drug is practically water-insoluble. ${ }^{8}$ However, solid dispersions of piroxicam by some water-soluble carriers were found to enhance drug dissolution. ${ }^{9-12}$ Of the amphiphilic carriers, which have attracted significant attention in pharmaceutical fields, are pluronic block copolymers. They are (poly (ethylene oxide)-block-(poly (propylene oxide)-block poly(ethylene oxide), $\mathrm{EO}_{\mathrm{m} / 2}-\mathrm{PO}_{\mathrm{n}}{ }^{-}$ $\mathrm{EO}_{\mathrm{m} / 2}{ }^{13-15}$ Pluronic polyols are chemically similar but physicochemically diverse surfaceactive agents composed of polymers of hydrophilic polyoxyehylene and lipophilic polyoxypropylene. Many researches have focused on these copolymers as transdermal delivery systems utilizing its gelling properties in gels formation. ${ }^{16-18}$

The aim of this article was to formulate and to study the interaction of piroxicam and different grades of pluronics, namely PL-F-68, PL F-98 and Pl-F-127 using different tools such as infrared spectroscopy (IR), X-ray diffractometry (XRD) and differential scanning calorimetry (DSC). The stability test for 1:1 solid dispersion of PL-F-98 was carried out and detected using (DSC). Phase diagram of solid dispersion of piroxicam and PL F-98 was studied at different weight ratios. The solubility of the drug in different pluronics was carried out, also the dissolution of the drug from its solid dispersion as well as its physical mixture was investigated.

\section{EXPERIMENTAL}

\section{Materials and methods}

Piroxicam was kindly donated by Sedico, Egypt. The characteristics of the Pluronic block copolymers Pl. F-68 \& Pl. F-127 (BASF, Aktiengesellschaft, Ludwigshafen, Germany) and Pl. F-98 (C.H.Erbsloeh, Krefeld, Germany) were presented in Table 1. All other chemicals used were of analytical reagent grade.

\section{Preparation of solid dispersions}

Solid dispersions containing piroxicam and each copolymer in different weight ratios (1:1, 1:3 and 1:5 drug: polymer) were prepared employing solvent evaporation method. The dug was dissolved in methylene chloride and the copolymer in a least amount of ethanol. The two solutions were mixed together and the organic solvents were removed by evaporation at room temperature with the aid of gentle stirring. The dried mass was pulverized, sieved and the $100-250 \mu \mathrm{m}$ particle size fractions were used in this study. The samples were kept in a desiccator till further investigations. Physical mixtures of piroxicam and different pluronics were prepared in the same ratios as in solid dispersions by gentle mixing for the drug and copolymer in a mortar.

\section{Infrared spectroscopy (IR)}

IR spectra were recorded with an IR spectrophotometer (IR-470, Shimadzu, Japan) by $\mathrm{KBr}$ disc method. The samples were scanned over the range of 4000 to $400 \mathrm{~cm}^{-1}$.

\section{Powder x-ray diffraction}

The x-ray diffraction (XRD) patterns of the samples were obtained using an automated X-ray diffractometer (Model FW 1700 series, Philips, Netherlands) using $\mathrm{CuK}_{\alpha}$ radiation $\left(\mathrm{CuK}_{\alpha}=1.5418 \AA\right)$, voltage $40 \mathrm{kV}$; current 30 $\mathrm{mA}$ and scanning speed $10 \mathrm{~mm} / \mathrm{sec}$.

\section{Thermal analysis}

Differential scanning calorimetry (DSC-50 Shimadzu, Japan) was used under the following conditions: sample weight $3-5 \mathrm{mg}$, scanning rate $10 \% \mathrm{~min}, \mathrm{~N}_{2}$ purge $30 \mathrm{ml} / \mathrm{min}$. The samples were heated in hermetically sealed aluminum pans in the temperature range $25-250^{\circ}$. Thermal analysis data were obtained using a TA 50I PC system with Shimadzu software programs.

\section{Stability test}

Solid dispersion of pluronic F-98 prepared at $1: 1$ weight ratio was stored at $40^{\circ}$ for 40 weeks. The melting point and the heat of fusion $(\mathrm{J} / \mathrm{g})$ were determined every 2 weeks for 40 weeks and at the end of study using (DSC). 
Table 1: Characteristics of the pluronic block copolymers used in this study

\begin{tabular}{|l|c|c|c||}
\hline & Pluronic F-68 & Pluronic F-98 & Pluronic F-127 \\
\hline Generic Name & Poloxamer 188 & Poloxamer 288 & Poloxamer 407 \\
\hline Molecular weight & 8400 & 13000 & 12600 \\
\hline Average No of PO units & 29 & 44.8 & 65.2 \\
\hline Average No of EO units & 152.7 & 236.4 & 200.4 \\
\hline PO/EO ratio & 18.99 & 18.95 & 32.53 \\
\hline HLB & 29 & 28 & 22 \\
\hline
\end{tabular}

$\mathrm{PO}=$ Propylene oxide, $\mathrm{EO}=$ Ethylene oxide, $\mathrm{HLB}=$ Hydrophilic-lipophilic balance .

\section{Solubility study}

Solubility of the drug in water in presence of different concentrations of pluronics $(0.03$, $0.0625,0.125$ and $0.25 \mathrm{mM}$ ) was studied. Solubility was studied using the following procedure: an excess amount of piroxicam powder was added to $10 \mathrm{ml}$ of each solution in a $120 \mathrm{ml}$ glass stoppered bottle. The bottles were firmly closed and placed into the mechanical shaking water bath (SBS Instruments, Germany) previously adjusted at $37^{\circ} \mathrm{C} \pm 0.5$ for $24 \mathrm{~h}$. One $\mathrm{ml}$ aliquot sample was withdrawn by pipette and diluted to an appropriate volume with simulate gastric secretion $\mathrm{pH}$ 1.2. The absorbance was measured at $331 \mathrm{~nm}$ (UV. 1601- Shimadzu Co. Japan) against blank. The concentration of the drug was calculated.

\section{Dissolution rate studies}

The dissolution rate of the drug from the prepared solid dispersions and some selected physical mixtures as well as the untreated drug was studied using USP dissolution apparatus (Erwecka Co., Germany). Accurately weighed amount equivalent to $10 \mathrm{mg}$ of piroxicam were dispersed over the dissolution medium $(900 \mathrm{ml}$ of simulate gastric secretion $\mathrm{pH} 1.2$ ) at $37^{\circ}$ \pm 0.5 which immediately stirred at $50 \mathrm{rpm}$. At time intervals, five $\mathrm{ml}$ sample was withdrawn and diluted to suitable volume with the dissolution medium and the absorbance was measured at $331 \mathrm{~nm}$ against blank. Equal volume of fresh dissolution medium, was replaced into the dissolution medium to keep the volume of the dissolution medium constant. The experiment was carried out in duplicate and the average of absorbance was calculated.

\section{RESULTS AND DISCUSSION}

IR spectra for piroxicam, pluronics, physical mixtures and solid dispersions were displayed in Figs. 1-3. The most characteristic bands of PXM are the band of N-H and enolic $\mathrm{O}-\mathrm{H}$ stretching at $3385 \mathrm{~cm}^{-1}$ for the needle form, $3330 \mathrm{~cm}^{-1}$ for the cubic form and the band for amid carbonyl stretching at $1637 \mathrm{~cm}^{-1}$. These values were comparable with those reported by Mihalic and Vrecer et al. ${ }^{19-20}$ The IR spectra of pluronic copolymer displayed a broad peak at 3355-3610, 3250-3625, 3245$3745 \mathrm{~cm}^{-1}$ for Pl. F-68, Pl. F-98, Pl. F-127 respectively. This broad peak did not prevent the appearance of $\mathrm{N}-\mathrm{H}$ or $\mathrm{O}-\mathrm{H}$ stretching vibration of piroxicam at $3395 \mathrm{~cm}^{-1}$. The position of the characteristic peaks for both of physical mixtures or solid dispersions are not affected but show broading and weakening in the higher ratio of the copolymer. This could be due to the dilution effect caused by copolymer. The results of IR spectra suggested the absence of interaction between piroxicam and pluronic copolymers. This result was in consistent with Fernandez et al. ${ }^{9}$ who found no chemical interaction between piroxicam and PEG 4000, which possess similar groups of pluronics.

The XRD patterns of PXM, Pluronics (Pl. F-68, Pl. F-98 and Pl. F-127), PMs and SDs were depicted in Figs. 4-6. PXM was a highly crystalline powder with characteristic diffraction peaks appeared at a diffraction angle of $2 \theta$ at 8.99, 15.75, 19.73, 22.99 and $25.84^{\circ}$. These values were comparable with those reported for needle form of piroxicam ${ }^{19}$ $(9.18,15.78,19.46,22.85,25.22)$. The pluronic copolymers produced two characteristic peaks 


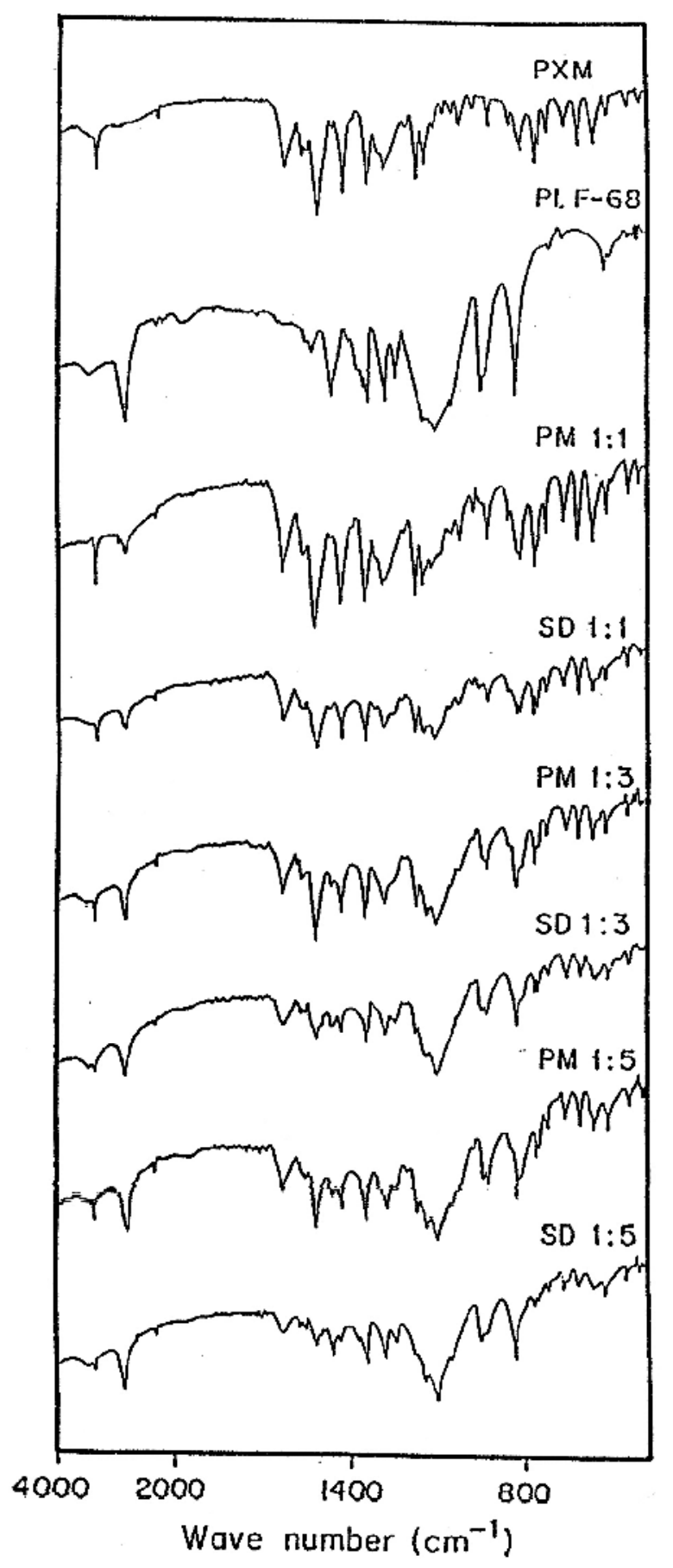

Fig. 1: Infrared spectrograms of piroxicam (PXM), pluronic F-68 (Pl. F-68), physical mixture $(\mathrm{PM})$ and solid dispersion (SD) in different drug polymer ratios.

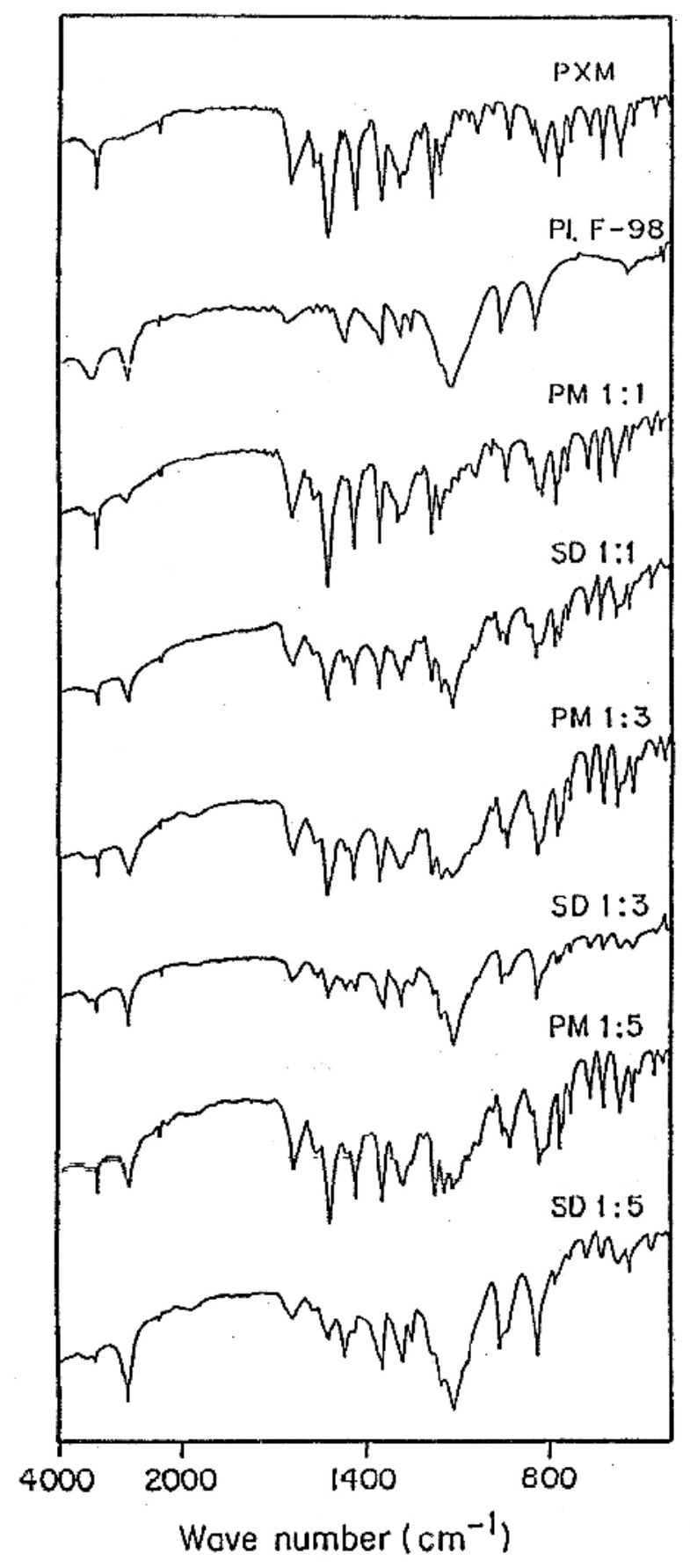

Fig. 2: Infrared spectrograms of piroxicam (PXM), pluronic F-98 (Pl. F-98), physical mixture (PM) and solid dispersion (SD) in different drug polymer ratios. 


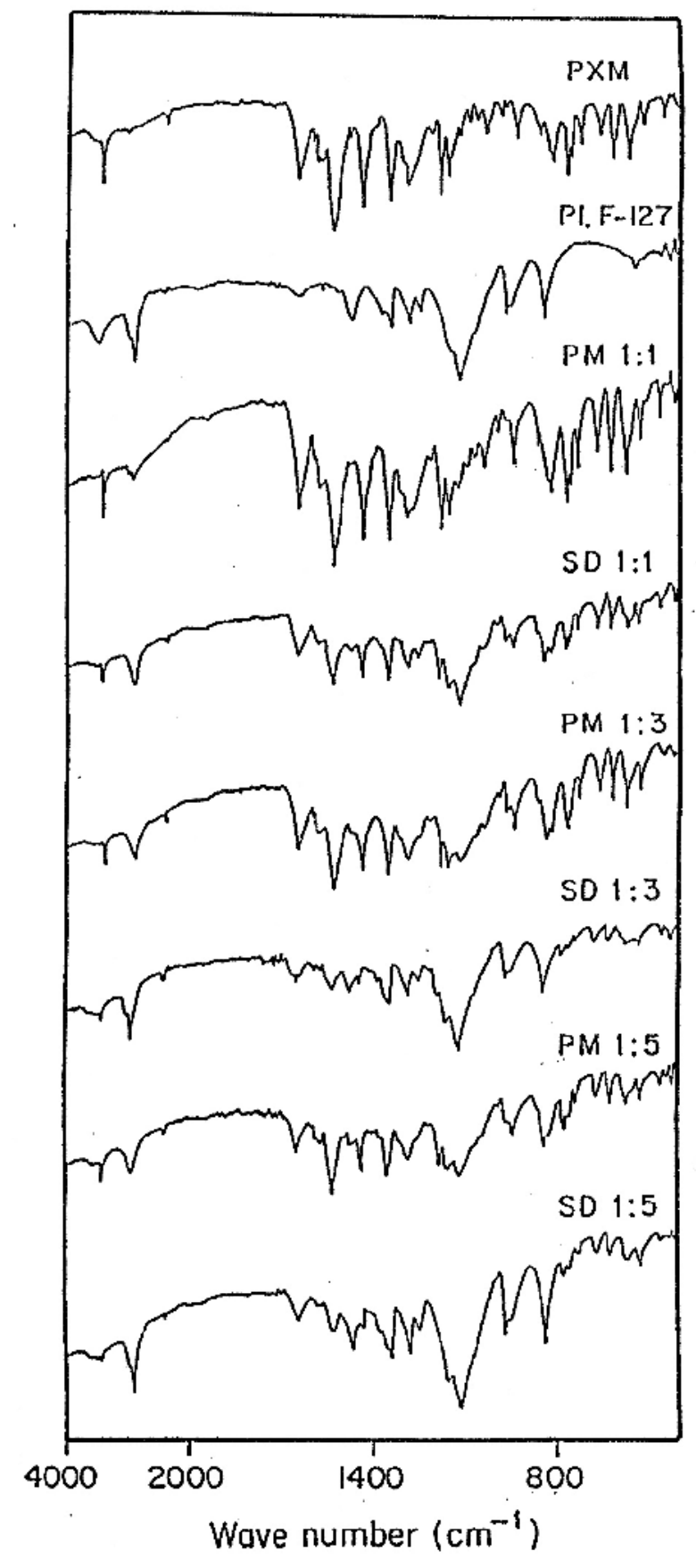

Fig. 3: Infrared spectrograms of piroxicam (PXM), pluronic F-127 (Pl. F-127), physical mixture (PM) and solid dispersion (SD) in different drug polymer ratios.

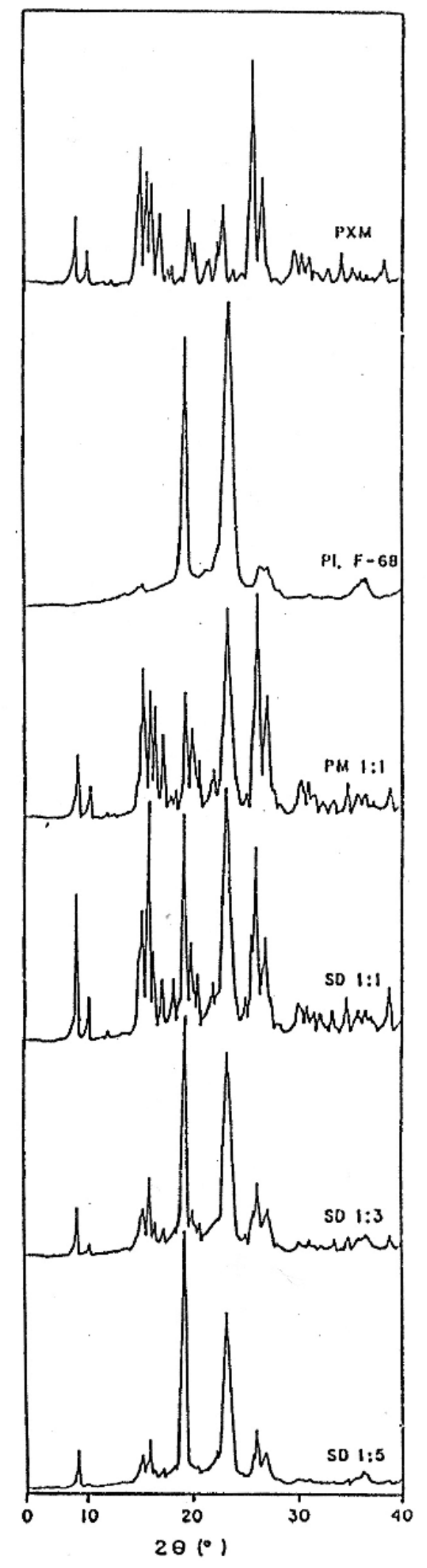

Fig. 4: X-ray diffraction patterns of piroxicam (PXM), pluronic F-68 (P1. F-68), physical mixture (PM) and solid dispersion (SD) in different drug polymer ratios. 


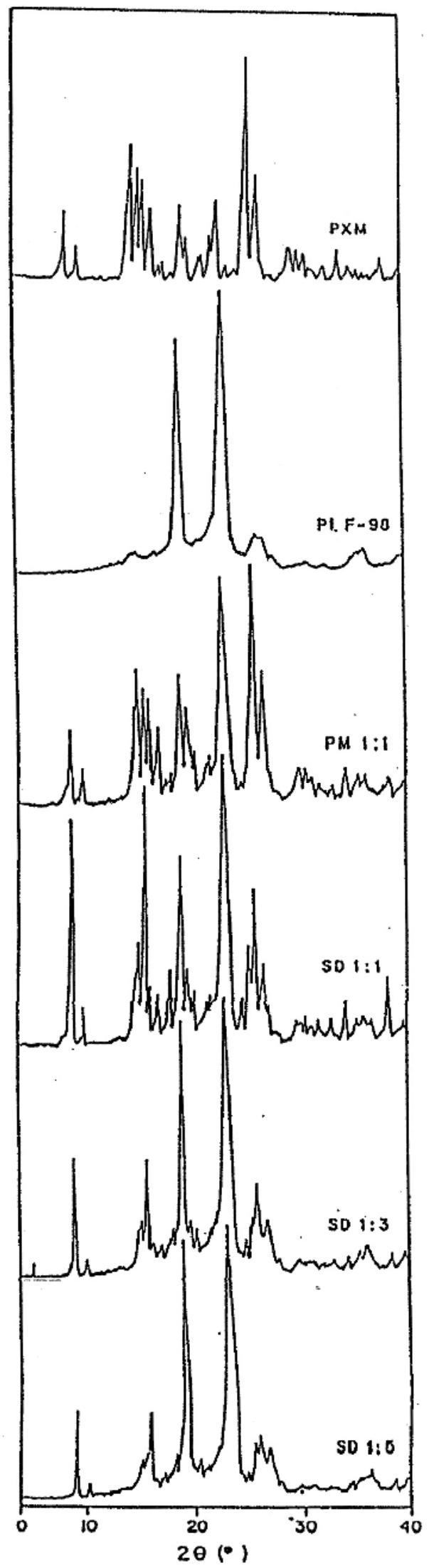

Fig. 5: X-ray diffraction patterns of piroxicam (PXM), pluronic F-98 (Pl. F-98), physical mixture (PM) and solid dispersion (SD) in different drug polymer ratios.

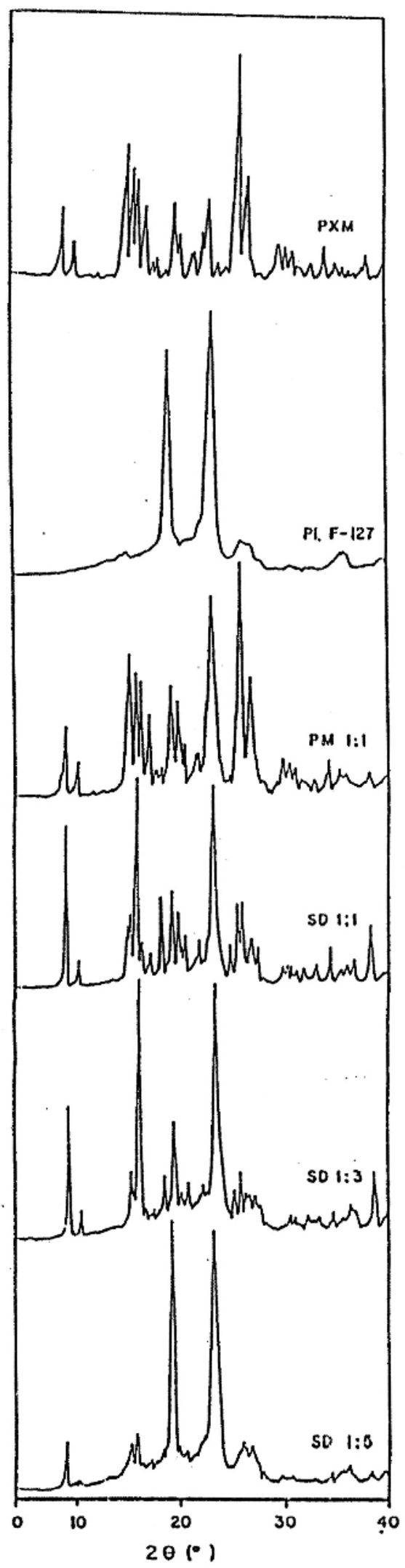

Fig. 6: X-ray diffraction patterns of piroxicam (PXM), pluronic F-127 (Pl. F-127), physical mixture (PM) and solid dispersion (SD) in different drug polymer ratios. 
of high intensity at $19.13,23.24^{\circ} ; 19.10$, $23.21^{\circ} ; 19.13,23.25^{\circ}$ for Pl. F-68, Pl. F-98 and Pl. F-127 respectively. The intensity of the major peaks of drug in case of SDs (1:1 ratio) was less than that of PMs prepared at the same ratio. The decrease in the intensity of PXM's major peaks in SDs was in the following order: Pl. F-68< Pl. F-98 <Pl. F-127. This difference could be attributed to the difference in lipophilicity and molecular weight of pluronics (Table 1), which might affect the dispersibility of PXM as well as the degree of it's mixing with the copolymer. The nonpolar hydrophobic moiety might promot solid solution formation. ${ }^{21}$ In contrast, Yuasa et $a .^{22}$ found that the dispersibility of flurbiprofen (FP) in the SD of hydroxypropyl cellulose (HPC) is higher with increasing HPC composition ratio. By increase in copolymer concentration, the piroxicam diffractograms were almost completely masked by the signals from the copolymer. The results indicated that, the less of crystalline properties of the drug or the drug may be found in a solid solution state at low concentration.

The DSC thermograms of PXM, Pluronics, PMs and SDs were shown in Figs. 79. Piroxicam showed a single fusion endotherm with a melting point at $201.5^{\circ}$. The melting points of pure polymers were $51.8,56.0,53.2^{\circ}$ for Pl. F-68 (Fig. 7), Pl. F-98 (Fig. 8), Pl. F-127 (Fig. 9), respectively. All PMs and SDs exhibited sharp endothermic peaks due to the fusion of pluronics at the same position as the pure copolymers. This revealed the existence of pluronics in the crystalline state that was in consistent with the appearance of the diffraction peaks in the corresponding XRD patterns. The melting of PXM was observed in the physical mixtures and in the SDs at 1:1 ratios as broad endotherms at a melting point less than that of pure drug.

The peak of PXM in SDs, prepared at 1:1 ratio, occurred at $191.7,192.6,193.9^{\circ}$ for Pl. F68, Pl. F-98, Pl. F-127 respectively. The disappearance of PXM melting endotherms was observed in all PXM-pluronic solid dispersions of higher than 1:1 ratio. The results might suggest that, the drug is much less crystalline or it is in amorphous form. Moreover, physical mixtures of PXM and different types of pluronics showed the same thermal behavior as the solid dispersions of the same compositions. These similarities between the DSC data of solid dispersion and the physical mixture indicated the absence of the chemical interaction between PXM and this copolymer. Yuasa et $a .^{22}$ reported that the reason for the disappearance of the endothermic peak in the PMs was presumably that the crystalline state of drug (flurbiprofen) became the amorphous state because of the enhancement in molecular mobility during the heating process.

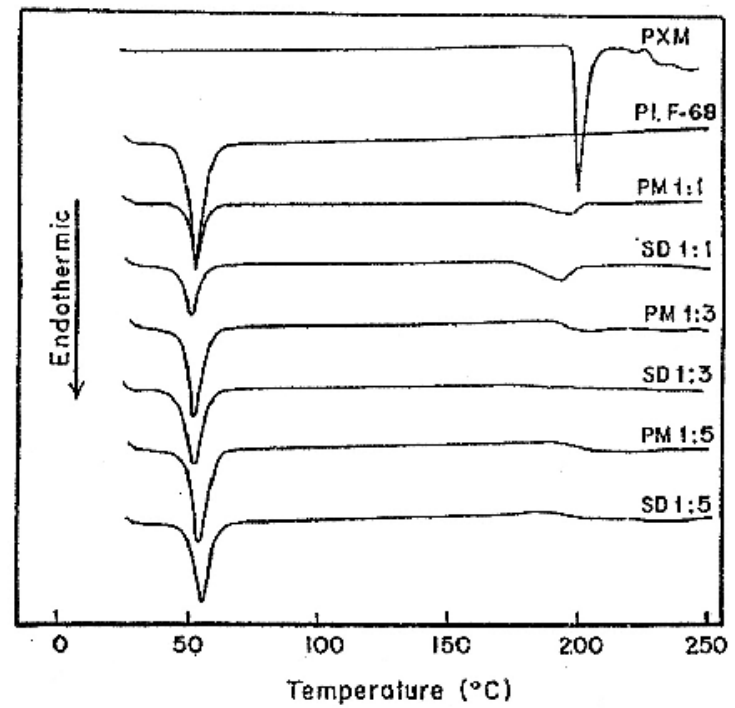

Fig. 7: DSC thermograms of piroxicam (PXM), pluronic F-68 (Pl. F-68), physical mixture (PM) and solid dispersion (SD) in different drug polymer ratios.

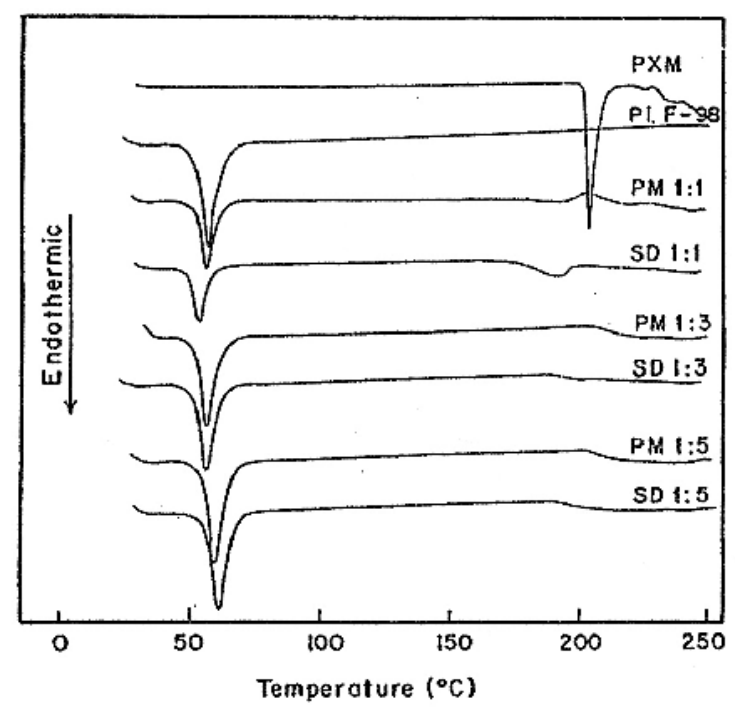

Fig. 8: DSC thermograms of piroxicam (PXM), pluronic F-98 (Pl. F-98), physical mixture (PM) and solid dispersion (SD) in different drug polymer ratios. 


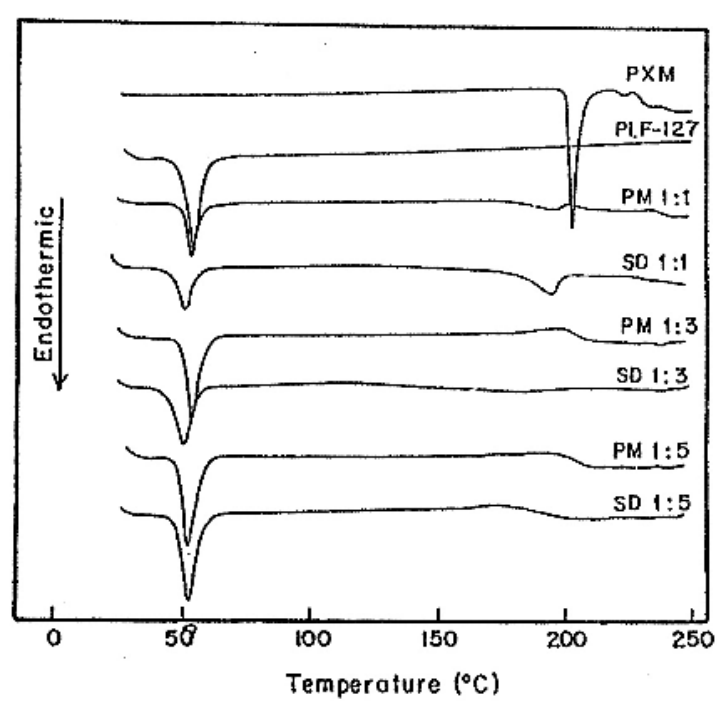

Fig. 9: DSC thermograms of piroxicam (PXM), pluronic F-127 (Pl. F-127), physical mixture (PM) and solid dispersion (SD) in different drug polymer ratios.

Construction of phase diagram of a drug and its prospective carrier from thermal analysis data is generally regarded as an important preliminary exercise in evaluating solid dispersion. ${ }^{23}$ Thermograms of various SDs prepared at different concentrations of $\operatorname{PXM}(0,20,40,50,60,80,100 \% \mathrm{w} / \mathrm{w})$ was used to construct PXM: Pl. F-98 binary phase diagram (Fig. 10). The resulting diagram showed that with an increase in the copolymer concentration, the melting point peak was decreased. Fourty percent w/w PXM gave an endothermic peak with a melting point $190.2^{\circ}$, while the concentrations less than $40 \% \mathrm{w} / \mathrm{w}$ gave no peak (Fig. 8). That means, the solidstate solutions were obtained if the concentration of PXM was less than $25 \%$ in the mixture. The heat of fusion as a function of the weight percent of PXM: Pl. F-98 was calculated and was plotted in Fig. $11 .^{24}$ The plotted heat of fusion values were the corrected ones $\left(\Delta \mathrm{H}_{\text {corr }}\right)$ and were obtained using the following formula:

$\Delta \mathrm{H}_{\text {corr }}=\left(\Delta \mathrm{H}_{\mathrm{obs}} / \% \mathrm{PXM}\right.$ in sample $) * 100 \ldots(1)^{24}$

Fig. 11 shows that, there is a linear relation between the composition of the solid dispersion and the heat of fusion values $(\mathrm{r}=0.988)$. The intercept on the abscissa did not cross the origin, however, the heat of fusion was zero, at less than $35 \% \mathrm{w} / \mathrm{w}$.

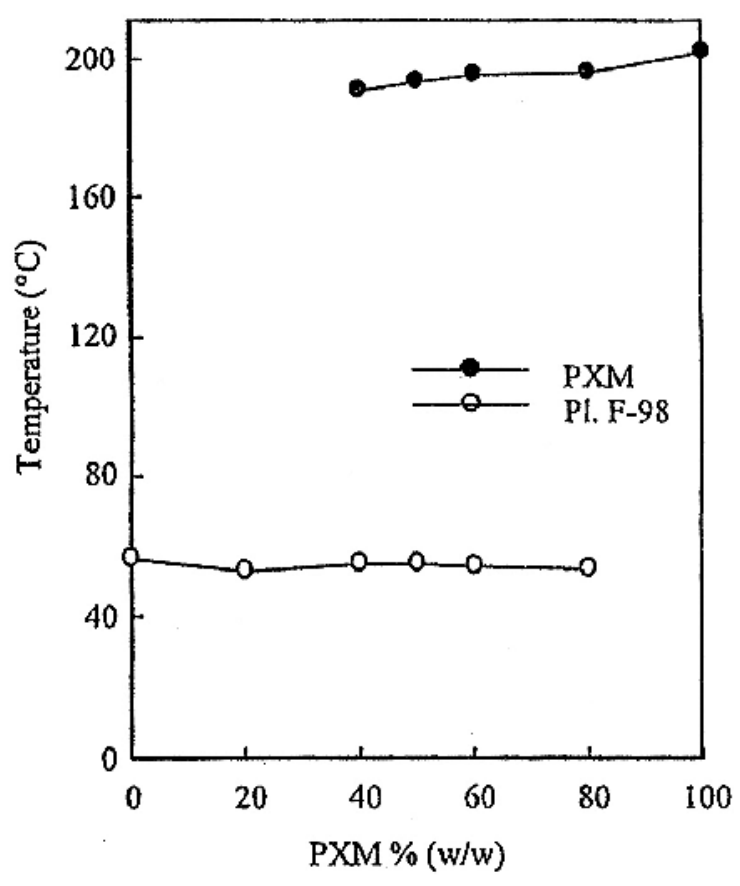

Fig. 10: Phase diagram for solid dispersion of piroxicam (PXM) in pluronic F-98 (Pl. F98).

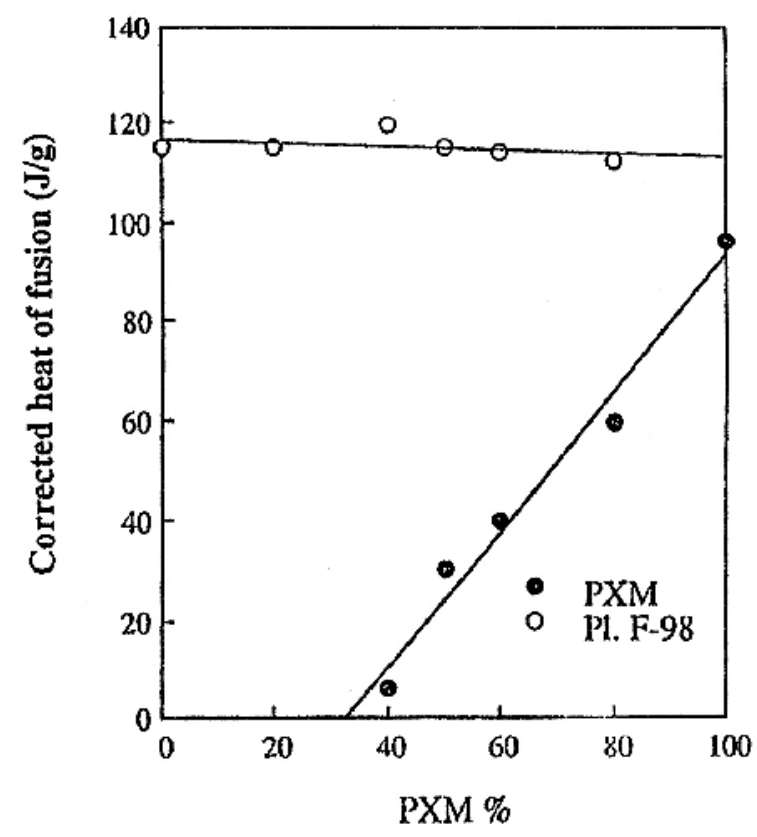

Fig. 11: Relation between piroxicam (PXM) concentration in solid dispersion of pluronic F-98 (Pl. F-98) and correct heat of fusion.

Figs. 10 and 11, indicated that, both of melting point and heat of fusion values did not change with the change of copolymer (Pl. F-98) concentrations which ascertained the previous conclusion that, pluronics in the crystalline 
state in the SDs in despite of its presence in low concentration $(20 \% \mathrm{w} / \mathrm{w}$ is lowest concentration used).

Table 2 demonstrated the melting point and the heat of fusion of PXM in the SD of pluronic F-98 prepared at 1:1 weight ratio after storage at $40^{\circ}$ for 40 weeks. The melting point of piroxicam in the solid dispersion was not changed. Moreover, the heat of fusion was barely affected. This indicated that, piroxicam in solid dispersion of pluronic F-98 was stable during aging.

\section{Solubility of piroxicam in pluronic solutions}

Different concentrations of different pluronics were studied for their solubilizing effect on piroxicam. The solubilization of piroxicam in different pluronic solutions at $37^{\circ}$ is shown in Figure 12. The tested concentrations were used above their respective critical micelle concentration (CMC). The solubility of the drug in these solutions was increased linearly by increasing the pluronic concentration.

The solubilization of a slightly watersoluble compound can be treated in terms of association equilibrium between the solutes and the micelles in a micellar solution ${ }^{25}$ Thus:

$\mathrm{D}_{\mathrm{w}}+\mathrm{M} \stackrel{\mathrm{Km}}{\longleftarrow} \mathrm{D}_{\mathrm{m}}$
Where $D_{m}$ and $D_{w}$ are the drug solubilized in the micellar and bulk water, respectively. $\mathrm{M}$ is the micellar concentration and $\mathrm{km}$ is the distribution coefficient of the drug between the micellar and the bulk aqueous phases.

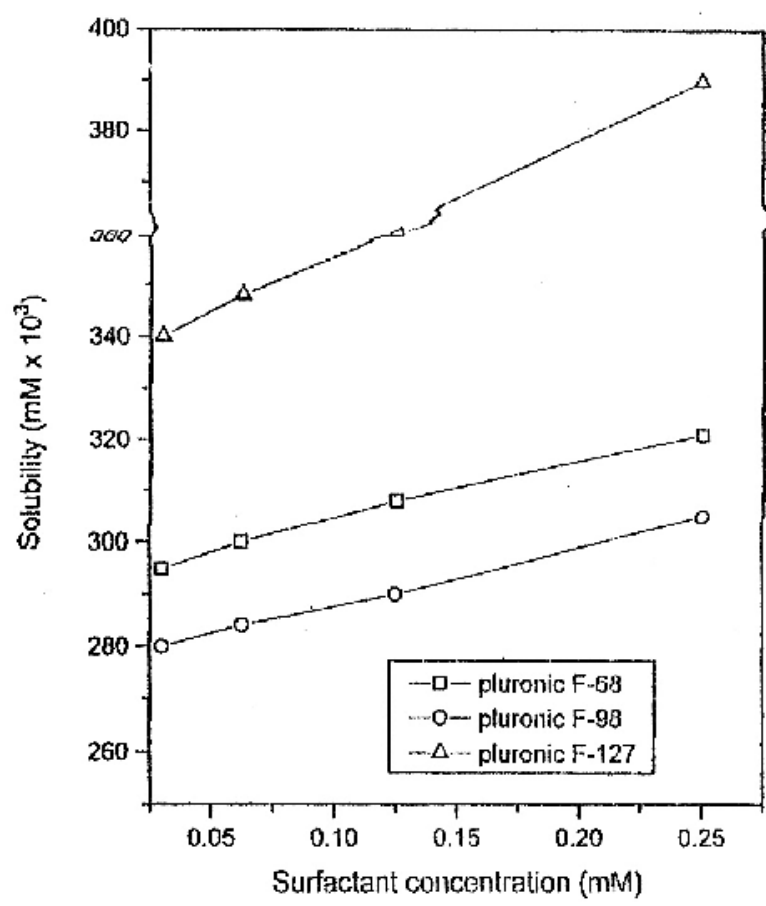

Fig. 12: Solubility or piroxicam in aqueous micellar solution of different pluronics at $37^{\circ}$.

Table 2: Melting points (m.p; $\left.{ }^{\circ}\right)$ and heat of fusion $(\Delta \mathrm{H} ; \mathrm{J} / \mathrm{g})$ for piroxicam and pluronic F-98 after storing their solid dispersion, prepared at 1:1 ratio, for 40 weeks at $40^{\circ}$.

\begin{tabular}{|c|c|c|c|c||}
\hline \multirow{2}{*}{$\begin{array}{c}\text { Storage time } \\
\text { (Weeks) }\end{array}$} & \multicolumn{2}{|c|}{ Pluronic F-98 peak } & \multicolumn{2}{c||}{ Piroxicam peak } \\
\hline 0 & $\mathrm{~m} . \mathrm{p}\left(^{\circ}\right)$ & $\Delta \mathrm{H}(\mathrm{J} / \mathrm{g})$ & $\mathrm{m} . \mathrm{p}\left(^{\circ}\right)$ & $\Delta \mathrm{H}(\mathrm{J} / \mathrm{g})$ \\
\hline 2 & 51.9 & 50.66 & 191.0 & 15.24 \\
\hline 4 & 50.3 & 53.42 & 192.5 & 16.49 \\
\hline 6 & 54.9 & 54.90 & 192.6 & 15.16 \\
\hline 8 & 54.6 & 60.77 & 192.2 & 18.41 \\
\hline 40 & 49.4 & 54.22 & 191.3 & 15.07 \\
\hline & 55.3 & 60.3 & 190.1 & 16.35 \\
\hline
\end{tabular}


Accordingly, the following relationships were drived:

$$
\mathrm{K}_{\mathrm{m}}=\mathrm{S}_{\mathrm{m}} / \mathrm{S}_{\mathrm{w}} \mathrm{M} \ldots \ldots \ldots \ldots \ldots
$$

and $S_{t}=S_{m}+S_{w}=S_{w}+K_{m} S_{w} M \ldots \ldots$.

Where $S_{\mathrm{m}}$ and $S_{\mathrm{w}}$ are the solubility of the drug in the micellar and bulk aqueous phase, respectively. $\mathrm{M}$ is the molar concentration of the pluronic and St is the total molar solubility of the drug in the micellar solution. Therefore, a plot of St versus $M$ will produce a straight line with an intercept of $S_{w}$ and a slope of $K_{m}$ $\mathrm{S}_{\mathrm{w}}$, which is the solubilizing capacity of the micelles. The slopes were determined by linear regression analysis. The distribution coefficient $\left(\mathrm{k}_{\mathrm{m}}\right)$ can be calculated from the slope $\left(\mathrm{k}_{\mathrm{m}} \mathrm{S}_{\mathrm{w}}\right)$ and $S_{\mathrm{w}}$.

Table 3 lists the solubilizing capacity of the tested pluronics and the distribution coefficient of the drug between micellar and bulk aqueous phases.

Table 3: Solubilizing capacity of different pluronics and the distribution coefficient of piroxicam between the micellar and aqueous phases.

\begin{tabular}{|c|c|c|}
\hline Pluronic & $\begin{array}{c}\text { Solubilizing } \\
\text { capacity } \\
\times 10^{2}\end{array}$ & $\begin{array}{c}\text { Distribution } \\
\text { coefficient } \\
(\mathrm{mM}) \times 10^{2}\end{array}$ \\
\hline Pluronic F-68 & 10.7 & 36.3 \\
\hline Pluronic F-98 & 10.3 & 37.0 \\
\hline Pluronic F-127 & 20.6 & 61.1 \\
\hline
\end{tabular}

The solubilization of piroxicam in different pluronic solutions Fig. 12, it was found that pluronic F-127 was more efficient as a solubilizer than pluronic F-68 and pluronic F98. In addition Pluronic F-127 exhibited a higher solubilization capacity and distribution coefficient than other pluronics.

\section{Dissolution rate studies}

Figures 13-15 show the dissolution profiles of piroxicam, its solid dispersions as well as the selected physical mixture with different pluronics block copolymer. From Figures 13-15, it was found that, the improvement of dissolution rates of piroxicam by different pluronics was ranked as Pluronic F-127 > F-68 $\geq$ F-98.

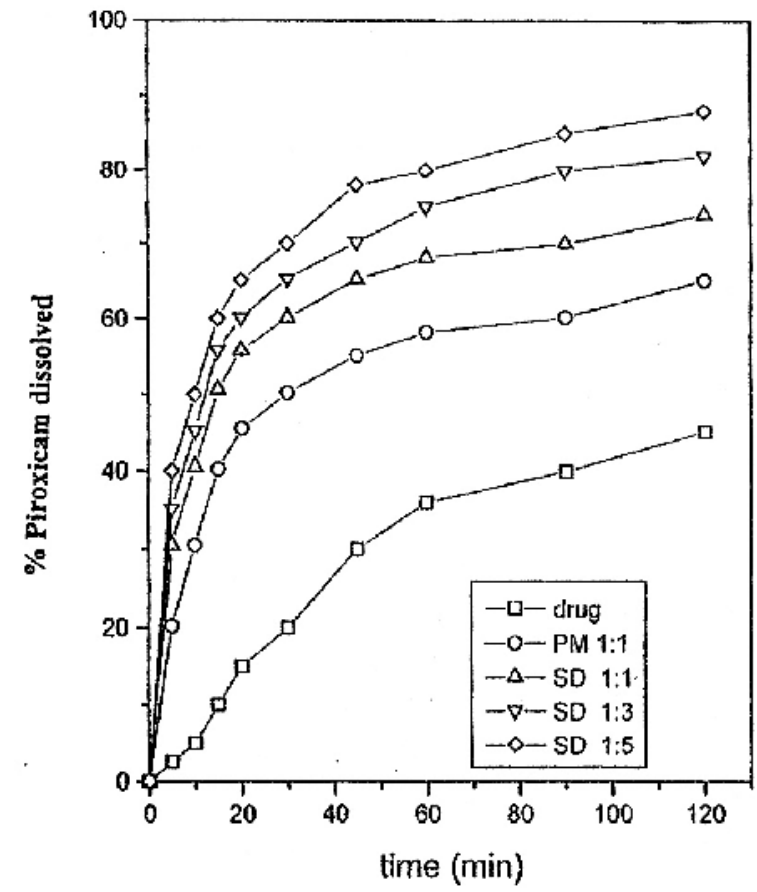

Fig. 13: Dissolution profile of piroxicam, its physical mixture and solid dispersion with pluronic F-98 at different drug polymer ratios.

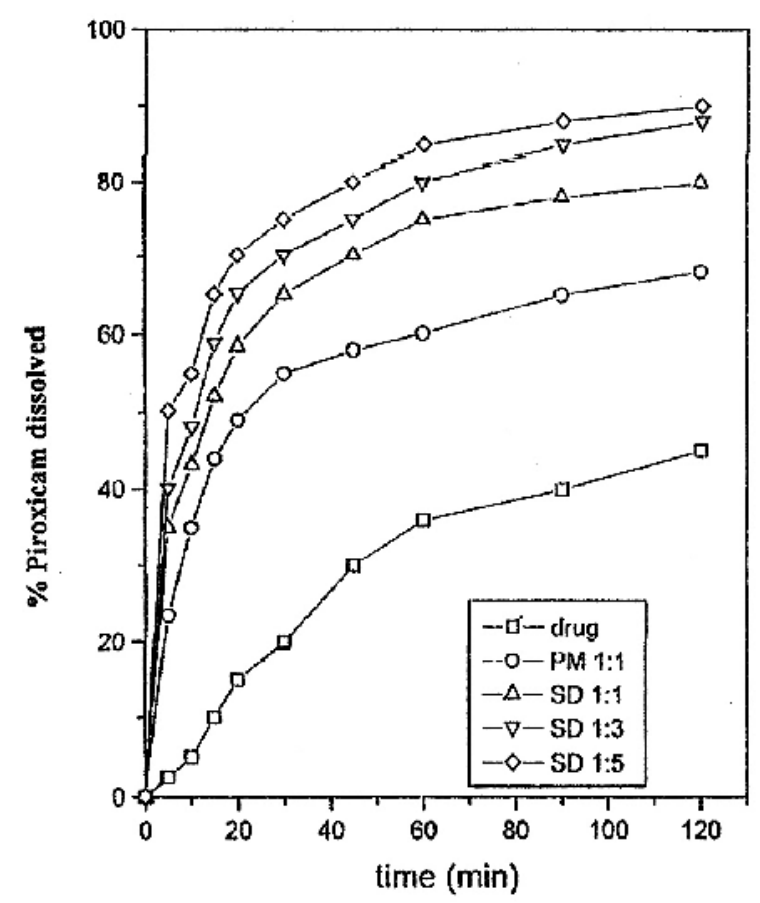

Fig. 14: Dissolution profile of piroxicam, its physical mixture and solid dispersion with pluronic F-68 at different drug polymer ratios. 


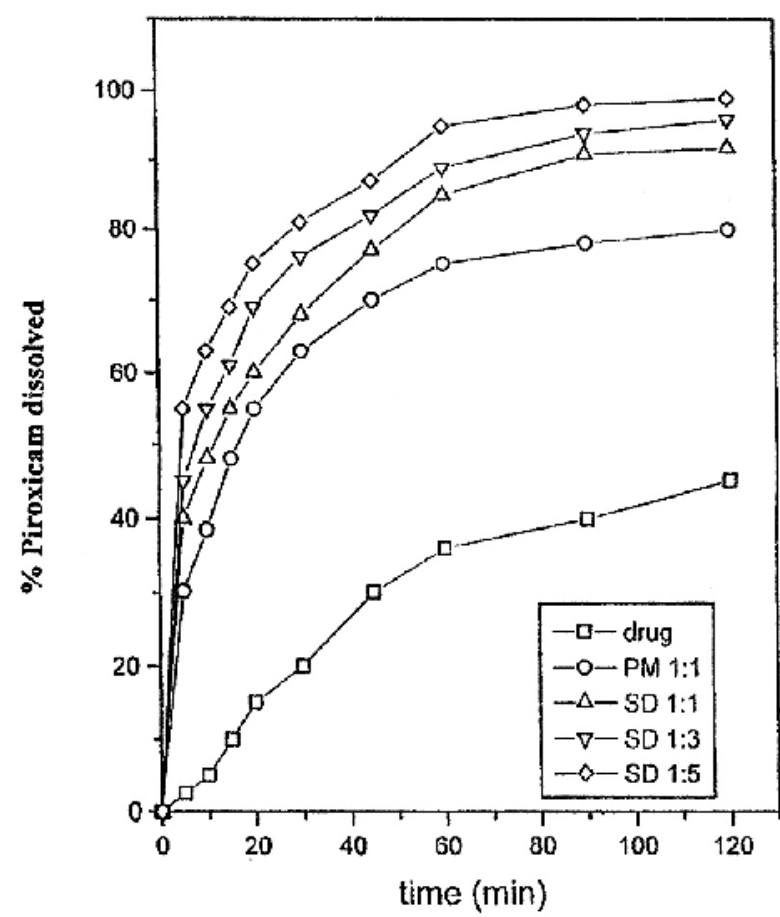

Fig. 15: Dissolution profile of piroxicam, its physical mixture and solid dispersion with pluronic F-127 at different drug polymer ratios.

It was found that, the physical mixtures exhibit a significant increase in the dissolution rate of the drug than the untreated drug. However, the solid dispersion displayed faster and higher dissolution rates than the corresponding physical mixture. Also, the dissolution rate of piroxicam was increase by increase the ratio of the carrier in the tested system. The dissolution rate of piroxicam from physical mixtures was greater than the drug itself. This may be explained in terms of the possible lowering of the surface tension of the medium by pluronics, resulting in better wetting of the drug surface. The improvement of the dissolution rate of the drug from its solid dispersion might be due to the conversion of its crystalline form into the amorphous one. ${ }^{26}$

In conclusion, IR spectroscopy, X-ray diffraction and DSC detected no chemical interaction between piroxicam and Pluronics. Pluronics keep their crystallinity in SD even at low concentration. Solid-state solutions were obtained for combinations containing less than $35 \%$ piroxicam. The solid dispersion for PXM was stable and no change in its form along 40 weeks at $40^{\circ}$. The aqueous solubility of the drug has been improved by using different pluronics. The dissolution rates of piroxicam was markedly improved either by physical mixture or the solid dispersion of different pluronics and this improvement can be ranked in a descending order as Pluronic F-127 > F-68 $\geq$ F-98.

\section{REFERENCES}

1- K. Sekiguchi, and N. Obi, Chem. Pharm. Bull., 9, 866 (1961).

2- M. Sugimoto, T. Okagaki, S. Narisawa, Y. Koida, and K. Nakajima, Int. J. Pharm., 160, 11 (1998).

3- K. P. R. Chowdary, R. O.Srinivasa, and S. K. Comm, Drug Dev. Ind. Pharm., 26, 1207 (2000).

4- S. D. Yoo, S-H. Lee, E. Kang, H. Jun, J-Y, Jung, J. W. Park, and K-H. Lee, Drug Dev. Ind. Pharm., 26, 27 (2000).

5- S. L. Law, W.Y. Lo, F.M.; Lin, and C. H. Chaing, Int. J. Pharm., 84, 161 (1992).

6- M. E. A. Dalmora, and A. G. Oliveira, Int. J. Pharm., 184, 157 (1999).

7- E. Swanepoel, W. Liebenberg, M. M. de Villiers, and T. G. Dekker, Drug Dev. Ind. Pharm., 26, 1067 (2000).

8- Parfitt, K., Martindale-the extra pharmacopoeia. 2002, $33^{\text {rd }}$ edition, the pharmaceutical press, London, p. 79.

9- M. Fernandez, I. C. Rodriguez, M. V. Margarit, and A. Cerezo, Int. J. Pharm., 84, 197 (1992).

10- M. Fernandez, M. V. Margarit, I. C. Rodriguez, and A. Cerezo, Int. J. Pharm., 98, 29-35 (1993).

11- V. Tantishaiyakul, N. Kaewnopparat, and S.Ingkatawornwong, Int. J. Pharm., 143, 59 (1996).

12- V. Tantishaiyakul, N. Kaewnopparat, and S. Ingkatawornwong, Int. J. Pharm., 181, 143 (1999).

13- A. V. Kabanov, and V. Y. Alakhov, Crit. Rev. Ther. Drug Carrier Syst., 19, 1 (2002).

14- P. Alexandridis, Colloid \& Interface Science, 2, 478 (1997).

15- E. Batrakova, S. Lee, S Li, A. Venne, V. Alakhov, and A. Kabanov, Pharm. Res., 16, 1373 (1999).

16- S. C. Shin, C. W. Cho, and H. K. Choi, Drug Dev. Ind. Pharm., 25, 273 (1999). 
17- S. C. Shin, E. Y. Shin, and C. W. Cho, Drug Dev. Ind. Pharm., 26, 563 (2000).

18- S. C. Shin, C. W. Cho, I. J. Oh, Int. J. Pharm., 193, 213 (2000).

19- M. Mihalic, In Florey, K., (Ed.), Analytical profiles of Drug Substances, Vol. 15, Academic press, London, 1986, pp. 509-531.

20- F. Vrecer, S. Srcic, and J. Smid-Korbar, Int. J. Pharm., 68, 35 (1991).

21- R. Kaur, D. J. W. Grant, and T. Eaves, J. Pharm. Sci., 69, 1317 (1980).
22- H. Yuasa, T. Ozeki, H. Takahashi, Y. Kanaya, and M. Ueno, Chem. Pharm. Bull., 42, 354 (1994).

23- W. L. Chiou, and S. Riegelman, J. Pharm. Sci., 60, 1281(1971).

24- T-T. Yang, and J. Swarbrick, J. Pharm. Sci., 75, 53 (1986).

25- J. T. H. Ong and E. Manoukian, Pharm. Res., 5, 704, (1988).

26- Y. Chiba, N. Kohri, K. Iseki and K. Miyazaki, Chem. Pharm. Bull., 39, 2158 (1991). 